\title{
Investigation of Multiple, Large Area EDS Detectors on an SEM Capable of Various Mounting Geometries for Optimal EDS Analysis.
}

\author{
D. Edwards ${ }^{1}$, N. Rowlands ${ }^{2}$, D. Guarrera ${ }^{1}$, and N. Erdman ${ }^{1}$, V. Robertson ${ }^{1}$, R. McLaughlin ${ }^{2}$. \\ 1. JEOL, USA Inc., 11 Dearborn Rd, Peabody, MA 01960 \\ 2. Oxford Instruments, Suite 150, 300 Baker Ave., Concord, MA 01742
}

It is common knowledge that collecting EDS data with dual large area SDD detectors from 180 degree opposing geometries has proven extremely beneficial with highly topographic samples [REF 1]. Traditionally, using high $\mathrm{kV}$, the high count rates achievable from today's SDD EDS detectors have been used to significantly reduce the time needed to acquire the statistical data necessary for X-ray analysis and mapping. Moreover, the use of low kV EDS for high spatial resolution analysis with minimized specimen damage has been recently gaining prominence [REF 2, 3]. When low $\mathrm{kV}$ microanalysis is performed, there can be a significant contribution from topography variations within the sample that can affect the quality and interpretation of the collected EDS data. Utilization of multiple large area EDS detectors can help alleviate some of these issues. In this paper the benefits of large area collection at low $\mathrm{kV}$ will be demonstrated on the next generation SEMs using conditions conducive to minimizing sample damage and contamination and to increasing spatial resolution. We will explore the benefits of multiple geometries with a variety of topographic samples. Special attention will be paid to detector positioning in these analyses and different geometries will be compared.

The JEOL JSM-IT300LV has the ability to mount multiple detectors and combine the data from 2, 3 or even 4 detector heads. This makes low $\mathrm{kV}$ EDS collection practical when combined with the near FE performance of this tungsten filament SEM with outstanding low $\mathrm{kV}$ performance. The combination of the Oxford Aztec system with dual X-Max ${ }^{\mathrm{N}} 80 \mathrm{~mm}^{2}$ detectors and the JEOL JSM-IT300LV SEM gives the ability to analyze highly topographic samples as well as highly beam sensitive samples at both high and low accelerating voltages.

We will show examples of EDS mapping of highly topographic materials with either random topography or 'linear' topography where the positioning of the detectors is taken into account to optimize results in all cases. For example, Fig. 1 shows an image of Si Ka EDS map of a highly topographic fracture surface taken at $15 \mathrm{kV}$ while varying the EDS detector position with respect to the specimen and adding multiple detectors to alleviate the topographic effect. We will also show examples of EDS mapping of a variety of specimens demonstrating the ability of this system to collect high spatial resolution data at low $\mathrm{kV}$ as well as observation and $\mathrm{X}$-ray analysis of beam sensitive materials without damage and contamination. The demonstrated instrumental configuration can provide a formidable tool for the analysis of rough surfaces at high speed as well as the analysis of beam sensitive samples at high spatial resolution using lower beam energies.

\section{References:}

[1] D.E Newbury and N.W.M Ritchie. Journal of Analytical Atomic Spectrometry 28.7 (2013): 973-988. [2] D.C. Bell and N. Erdman, in Low Voltage Electron Microscopy: Principles and Applications (2012): $1-30$.

[3] N. Rowlands et al. Microscopy and Microanalysis 15.S2 (2009): 548-549. 

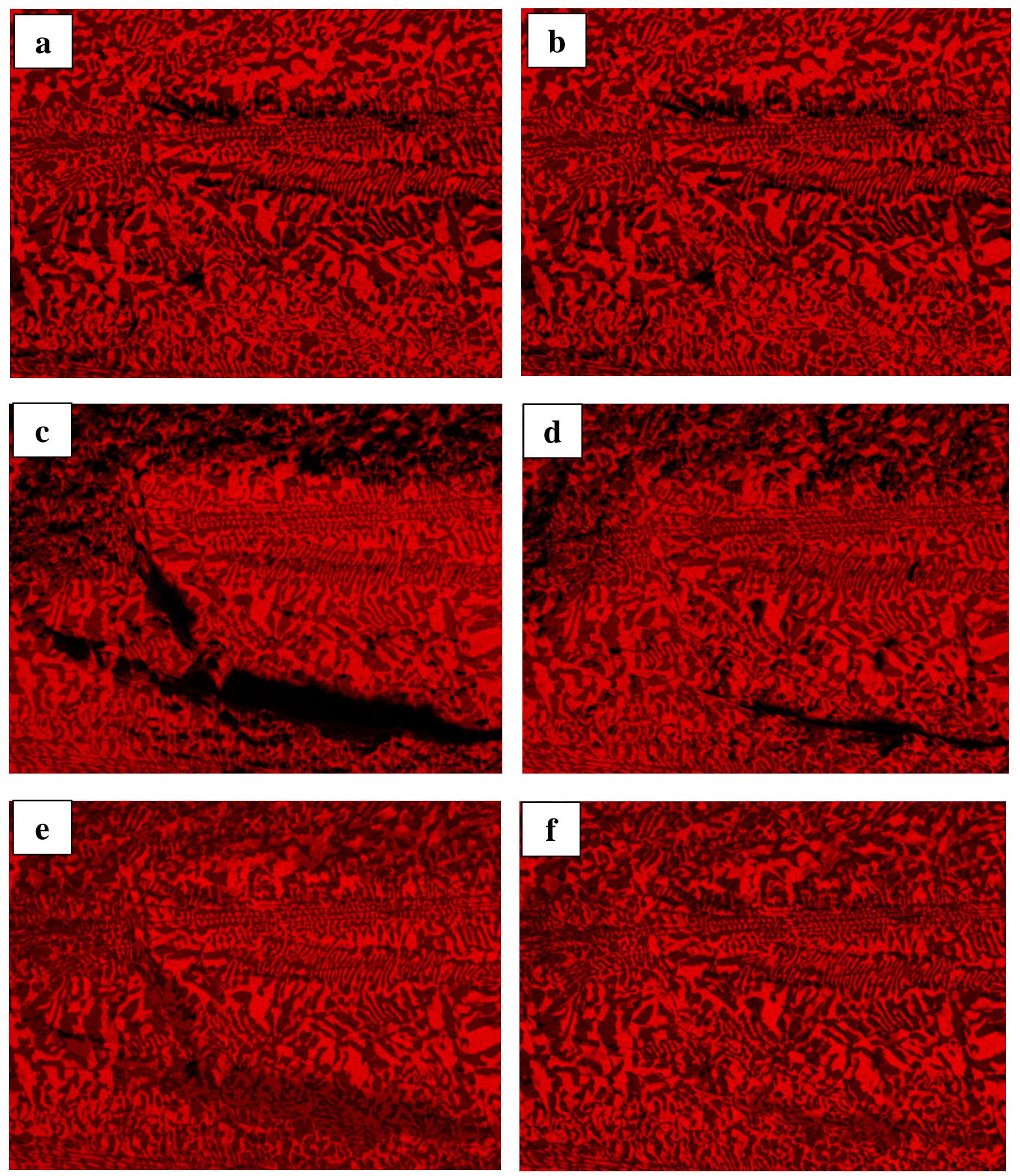

Figure 1. Silicon Ka EDS map of a highly topographic fracture surface taken at $15 \mathrm{kV}$ with: (a,b) one EDS detector mounted at the top of the image, (c) one EDS detector mounted at the bottom of the image (d) one EDS detector mounted toward the right of the image. (e) two EDS detectors mounted at the top and bottom of the image (f) two EDS detectors mounted at the top and right of the image. 